\title{
Zum Tod von Herrn Prof. Dr. Léon Wurmser
}

\author{
Ulrich Bahrke • Beata Hammerich • Beate Steiner
}

Online publiziert: 27. März 2020

(C) Springer Medizin Verlag GmbH, ein Teil von Springer Nature 2020

Ein wegweisender Wissenschaftler, einer der großen bedeutenden Psychoanalytiker aus der Generation der heutigen weisen Lehrer, Herr Prof. Dr. Léon Wurmser, ist am 15.02.2020 verstorben. Im Kreis seiner Familie ist er friedlich eingeschlafen, nachdem er vier Jahre lang durch die Folgen eines Hirnschlags an das Bett gefesselt war. Léon Wurmser ist 89 Jahre alt geworden. Am 17.02.2020 wurde er in Baltimore, Maryland, beigesetzt.

Viele in Europa lebende Fachkolleg*innen durften in den vergangenen Jahrzehnten von ihm lernen und ihn als offenen, undogmatischen Menschen, Lehrer, Wissenschaftler und Psychoanalytiker erleben. Sehr genau hinzuhören, einfühlsam zu verstehen und zu versuchen, den Patienten nicht blind für dessen Seele zu übergehen, sondern auf sein und unser Herz zu hören, sind wichtige Prinzipien, die wir im Austausch mit ihm immer wieder eindrucksvoll erleben durften. Wie seinen Patient*innen begegnete er auch uns Kolleg*innen mit großer Freundlichkeit und Respekt. In der Begegnung mit ihm zeigte sich immer wieder, in welch authentischer Weise er die Werte, die er vertrat - innere Freiheit, Wahrhaftigkeit und Ehrfurcht vor dem Leben - auch lebte. Für Léon Wurmser war es kein Zufall, sich sein Leben lang mit dem Phänomen des Tragischen zu beschäftigen: Er führte es auf sein jüdisches Erbe zurück, auf seine Zugehörigkeit zu einem Volke mit einer besonderen Kulturtradition und Bedrohtheit. Als wenige Kilometer von der deutschen Gren-

PD Dr. med. U. Bahrke

Neptunstr. 4, 8032 Zürich, Deutschland

Dipl.-Psych. B. Hammerich $(\bowtie)$

Landsbergstr. 15, 50678 Köln, Deutschland

E-Mail: Beata.Hammerich@web.de

B. Steiner

Liebigstr. 13a, 64293 Darmstadt, Deutschland

E-Mail: beate-steiner@t-online.de 
ze in Bülach aufgewachsenes und auch dort von Antisemitismus umgebenes Kind war ihm beides existenziell eingeschrieben. Es war für ihn insofern folgerichtig, sich sowohl mit dem Thema der Traumatisierung und seiner inneren Verarbeitung durch Schuld und Scham wie auch mit dem der unsichtbaren tragischen Konflikte immer wieder beschäftigen zu müssen; und es war ihm Verpflichtung, dem Traumatischen des Holocaust den Reichtum der jüdischen Kultur entgegenzusetzen. Nicht zuletzt daraus resultierte seine besondere Betonung der Phänomene der traumatischen Hilflosigkeit und der „Allmacht der Verantwortlichkeit“ sowie der Bedeutung des geduldigen feinfühligen Zuhörens im Sinn von Zeugenschaft.

Léon Wurmser verfügte über ein so reiches Wissen, wie wir es bei niemandem sonst erlebten, neben der Psychoanalyse und Psychiatrie vor allem in Philosophie, Judaistik, Weltgeschichte und Altertumswissenschaften. Und es war ihm eine geläufige Selbstverständlichkeit, in seinen Supervisionen Verknüpfungen mit Werken der Weltliteratur herzustellen, die, im Original gelesen zu haben, er auch uns aufforderte. Vor der Goethe-Gesellschaft zur „europäischen Seele“ zu sprechen, klang bei ihm nicht vermessen. Als er 2004 von der Humboldt-Universität Berlin die Ehrendoktorwürde verliehen bekam, sprach er über „Verstehen statt Verurteilen“ - sein Wissen und seine Grundhaltung verknüpfend.

In seinen Seminaren, Vorlesungen und Supervisionen wurde er nicht müde, mit dieser Grundhaltung sein Grundverständnis zu vermitteln, psychische Störungen, und eben auch schwere Neurosen und frühe Störungen, als Ausdruck von Konflikten zu betrachten, dabei Scham- und Schuldgefühle als den Kern einer Vielzahl von Neurosen herauszuarbeiten, sowie die zentrale Bedeutung des archaischen Über-Ich, von Ressentiment und Wiederholungszwang. Dabei hat er gerade auch in Supervisionen die enge Verbindung von Kindheitstrauma und Konflikt bei schweren Neurosen aufgezeigt und uns mit seinem Blick von außen früher als andere auf transgenerationale Weitergaben von Opfer- und Täterschicksalen bei uns und unseren Patienten aufmerksam gemacht. Anhand konkreter Falldarstellungen konnten wir so die gravierenden Auswirkungen tiefer Demütigung und Beschämung durch Erfahrungen seelenblinder und seelenmörderischer Erfahrungen nachvollziehen. Er konnte uns vermitteln, wie sich diese Traumatisierungen im Über-Ich im Einzelnen in der Destruktivität gegen das Selbst und gegen andere verfestigten. So konnte und kann die Brisanz dieser Problematiken, die Léon Wurmser auch anhand seiner ausführlichen Fallbeispiele, die er bei psychoanalytischen Kongressen, aber auch vor einem breiten psychotherapeutischen Kollegium wie dem der Lindauer Psychotherapiewochen sowie in seinen Büchern darlegte, uns manche tragische und bedrückende soziale Realität erklären: Denn ,je heftiger und bitterer das Ressentiment, basierend auf tiefer Demütigung und Scham, desto schärfer zerfällt die Weltsicht ins Absolute, in ein Entweder-oder; und umso verheerender ist die Rachsucht, die über den Einzelnen hinaus auch ganze Gesellschaften in gewaltige Ausschreitungen und Verwüstungen führen kann" (Steiner und Bahrke 2013, S. 10, 11).

Léon Wurmsers Wirken war keineswegs auf die USA und die deutschsprachigen Länder beschränkt. Neben der universitären Lehre in den Vereinigten Staaten fanden regelmäßige Lehr- und Fortbildungsveranstaltungen in zahlreichen europäischen Ländern, aber auch im ehemaligen Ostblock statt. So sind auch seine Verdienste um den internationalen wissenschaftlichen Austausch und Dialog außerordentlich 
groß. Seine zahlreichen Buchpublikationen, in vielen Sprachen veröffentlicht, werden nicht nur wegen ihres wissenschaftlich hohen Niveaus bei Fachkolleg*innen sehr geschätzt, sondern sind zudem thematisch breit gestreut. So hat Léon Wurmser u. a. in vielen seiner Beiträge die Formen und Strukturen des Über-Ich thematisiert und dieses Thema auch in die Verzweigungen philosophischer Erörterungen und kulturell-gesellschaftlicher Aspekte hinein dargestellt.

Léon Wurmser hat uns ein großes Lebenswerk hinterlassen, das für alle, die ihn kannten, mit seiner so überaus liebenswerten, humorvollen, neugierigen, geistreichen, kreativen und auch bescheidenen Person verbunden ist und in unserer Erinnerung bleiben wird.

Zürich, Köln, Darmstadt, März 2020

Ulrich Bahrke, Beata Hammerich, Beate Steiner

\section{Literatur}

Steiner B, Bahrke U (Hrsg) (2013) Der innere Richter im Einzelnen und in der Kultur - Klinische, soziokulturelle und literaturwissenschaftliche Perspektiven - Léon Wurmser zum 80. Geburtstag. Psychosozial Verlag, Gießen

Ulrich Bahrke Priv.-Doz. Dr. med., Facharzt für Psychiatrie und Psychotherapie FMH, Psychoanalytiker (DPV/IPA) und Lehranalytiker (DGPT) in eigener Praxis; Dozent am Freud Institut Zürich. Seit 2007 tätig am Sigmund-Freud-Institut in Frankfurt am Main, bis 2015 Leiter der Institutsambulanz und Klinischer Leiter der LAC-Depressionsstudie. Vorstandsmitglied der Deutschen Gesellschaft für Psychoanalyse und Musik (DGPM).

Beata Hammerich Dipl.-Psych., Psychologische Psychotherapeutin, Psychoanalytikerin (DGPT) in eigener Praxis in Köln.

Beate Steiner Dipl.-Psych., Psychologische Psychotherapeutin, Psychoanalytikerin (DGPT), Psychotraumatherapeutin (DeGPT). Lehrpsychotherapeutin und -analytikerin, Supervisorin und Dozentin, Leiterin des Instituts „Arbeitskreis für Psychotraumatologie und Katathym Imaginative Psychotherapie“ in Darmstadt. 